\title{
Adjuvant, neoadjuvant, and experimental regimens in overcoming pancreatic ductal adenocarcinoma
}

\author{
Olga Wysocka, Julita Kulbacka, Jolanta Saczko \\ Department of Medical Biochemistry, Wroclaw Medical University, Wroclaw, Poland
}

Gastroenterology Rev 2016; 11 (3): 155-162

DOI: $10.5114 /$ pg.2016.61438

Key words: pancreatic cancer, epidemiology, treatment.

Address for correspondence: Julita Kulbacka, Department of Medical Biochemistry, Wroclaw Medical University, 10 Chałubińskiego St, 50-368 Wroclaw, Poland, phone: +48 71784 13 87, fax: +48 7178400 85, e-mail: julita.kulbacka@umed.wroc.pl

\begin{abstract}
Pancreatic cancer is one of the most aggressive and deadly malignancies. Despite better understanding of its biology and pathogenesis, contemporary treatment regimens are still insufficient. Along with the introduction of new treatment agents and combination therapy, the response rates are increasing, but these scores do not go with overall survival, and results are frequently conflicting. Therefore, contemporary medicine faces the challenge of expanding the knowledge base and practice on all grounds - pathology, factor risk, diagnosis, and finally surgical and palliative treatment of this disease. This paper provides a review of current adjuvant and neoadjuvant regimens and the role of experimental therapies in pancreatic ductal adenocarcinoma.
\end{abstract}

\section{Epidemiology}

Worldwide, over 200,000 people a year are diagnosed with pancreatic cancer. The greatest risk of morbidity (approximately 10 per 100,000 population per year) relates to the population of developed countries, where pancreatic cancer is the fifth most common cause of cancer death [1]. In the United States this type of cancer is currently the fourth leading cause of cancer death and it is estimated to become the second by 2030 [2].

Pancreatic ductal adenocarcinoma (PDAC) is a rapidly progressing and late diagnosed exocrine cancer. For this reason pancreatic cancer has the worst mortality rate and the lowest overall survival in all cancers [3]. Only $5 \%$ to $25 \%$ are eligible for resection, and even after RO resection median survival amounts only 12 and 20 months and the 5 -year survival does not exceed 20\% [4-6]. For patients with locally advanced and metastatic disease prediction is much worse with the 5 -year survival rates below $22 \%$ and $6 \%$, respectively [5]. At the moment of diagnosis as many as 50\% patients are burdened with metastatis and their median survival time is only 3-6 months $[7,8]$.

There is growing evidence suggesting a significant impact of internal and environmental factors on pancreatic cancer occurrence. Recent literature reports confirm that the most important factors include: family history, smoking, and diabetes $[9,10]$. High morbidity among older people suggests that pancreatic carcinoma is strongly associated with age. Diet and healthy lifestyle also seem to be significant. The results of Li et al. show that overweight or obesity during early adulthood is linked with greater risk of pancreatic cancer. The same studies indicate that obesity at older age in patients with pancreatic cancer is associated with a lower overall survival [11]. There is also some 'limited' or 'probable' evidence that dietary elements such as fat, vitamin D, or folate may be associated with pancreatic cancer [12]. Also, processed meat consumption and heavy alcohol drinking are considered to be factors increasing the risk of pancreatic cancer occurrence $[13,14]$. The effect of alcohol intake seems to be age-dependent: the strongest association concerns very-early-onset pancreatic carcinoma [9].

Up to $4-16 \%$ of patients have a positive family history for pancreatic cancer [15]. Despite the lack of strong evidence, pancreatic cancer has been associated with several units such as breast cancer (due to germ-line mutations in BRCA1 and BRCA2 gene), PeutzJeghers syndrome, familial atypical multiple mole melanoma, and hereditary nonpolyposis colorectal cancer [15-17]. Research shows that also patients with hered- 
itary pancreatitis are at high risk of pancreatic cancer, even a few decades after the occurrence of inflammation. Admittedly, pancreatitis-associated gene mutations are not directly responsible for the development of PDAC; however, they provide a high-risk inflammatory environment for the accumulation of oncogenic mutations [18]. There is some evidence that hereditary pancreatitis may increase the PDAC risk by up to $40 \%$ and that the risk is much higher when pancreatitis is paternally inherited [19].

In conjunction with inflammation, alteration in bile flux and disorder of hormonal balance gallstones and cholecystectomy may also increase the risk of pancreatic cancer $[20,21]$. Therefore, cholecystectomy is considered to be both the cause and the symptom of pancreatic carcinoma.

\section{Contemporary regimens}

Depending on the progression of the disease there are various procedures employed in combating pancreatic ductal adenocarcinoma (Figure 1).

\section{Surgical treatment}

Less than $20 \%$ of patients are amenable to surgical resection, which is the only potentially curative treatment for pancreatic cancer [22]. Tumours that have not embraced the portal and superior mesenteric veins and not attacked the celiac or superior mesenteric artery roots are defined as unresectable. Also, local advancement and the presence of distant metastases are contraindications for this procedure [23]. Since the majority of tumours form in the head of the pancreas, pancreaticoduodenectomy (Whipple procedure) is a common strategy in pancreatic cancer treatment. Pylorus-preserving modification of this method, Tra-
verso-Longmire procedure, is also acceptable. Notwithstanding, expanded lymphadenectomy does not improve overall survival [24]. For body and tail cancer, a subtotal pancreatic left resection is recommended [25]. As a result of increasing safety of operations, recently the resection criteria are changing and there is a growing possibility of palliative resection for people with limited but incurable disease [26].

\section{Adjuvant therapy}

Since 5 -year survival reaches around $10 \%$ following resection, there is a clear need to improve this score by developing adjuvant therapy [27]. Currently, among first- and second-line therapy the most important role is played by fluoropyrimidine-based and gemcitabine-based chemotherapy.

\section{Chemotherapy}

\section{5-FU}

Fluorouracil (5-FU) is an antimetabolite with multidirectional activity, but in cancer treatment it primarily acts as an irreversible inhibitor of thymidylate synthase (TS). The mechanism of action is based on intracellular conversion of 5-FU to several active metabolites that preclude full RNA synthesis and the action of TS [28].

Until the mid-1990s, 5-FU delivery was the standard procedure for patients after resection or with unresectable tumour. However, the initial enthusiasm was muffled by subsequent trials. In 1991 Decaprio et al. reported a response rate below $10 \%$ in patients threatened with the combination of 5-FU and leucovorin [29]. Leucovorin is a reduced form of folic acid that enhances the effectiveness of 5-FU by stabilising the fluorouracil-TS complex. There have been many attempts to improve response rates by combining 5 -FU with other drugs

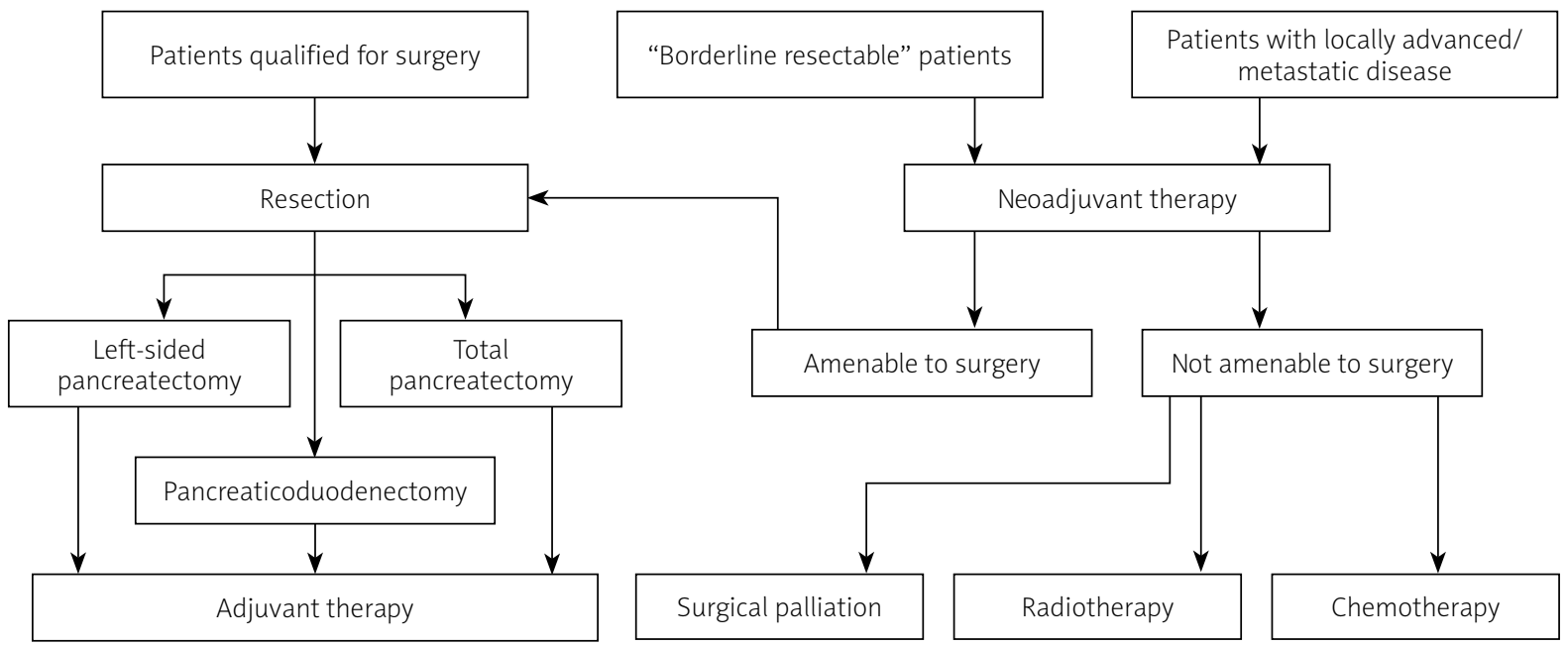

Figure 1. Treatment plan for pancreatic ductal adenocarcinoma in different stages of disease 
like Cytoxan, methotrexate, vincristine, or mitomycin C, but none brought significant benefit compared to 5 -FU monotherapy [30]. The most recent achievement in improving the efficiency and therapeutic index of orally administered 5 -FU is drug S-1. This fluoropyrimidine consists of tegafur, which is a prodrug of 5-FU, and it is supplemented by two inhibitors: dihydropyrimidine dehydrogenase inhibitor and orotate phosphoribosyltransferase inhibitor [31]. Among the precursors of 5-FU the greatest popularity is achieved by capecitabine. Results of chemoradiotherapy with capecitabine or 5-FU showed that higher intratumoural and lower systemic concentration of capecitabine result in significantly lower haematological and non-haematological toxicities compared to bolus 5-FU. Notwithstanding, overall response rates and median OS time remained at comparable levels [32].

\section{Gemcitabine}

The first drug that was proven to be more effective than 5-fluorouracil was gemcitabine, which along with 5-FU and capecitabine belongs to the group of antimetabolites acting as pyrimidine antagonists. In a randomised trial conducted on 126 patients with advanced symptomatic pancreas cancer nearly $24 \%$ of patients experienced clinical benefit response, whereas in patients receiving 5-FU this primary efficacy measure did not exceed $5 \%$. Superiority of gemcitabine-based treatment manifested also in median survival (5.65 and 4.41 months for gemcitabine and 5-FU, respectively) and survival rate at 12 months (18\% for gemcitabine vs. $2 \%$ for 5-FU) [33]. Based on this clinical benefit the Food and Drug Administration (FDA) approved gemcitabine monotherapy as first-line treatment for advanced pancreatic cancer [34]. The latest reports, however, seem to be conflicting.

The trial conducted by the European Study Group for Pancreatic Cancer (ESPAC) shows that gemcitabine treatment does not result in improved overall survival in comparison with fluorouracil plus folinic acid therapy. In this phase III trial, conducted on 1088 patients with PDAC after cancer resection, median survival for patients treated with 5-FU plus folinic acid was 23.0 months and for patients receiving gemcitabine it was less than 3\% (23.6) higher. Fourteen percent (97) of patients in the 5 -FU group had treatment-related serious adverse events in comparison to $7.5 \%$ (52) of events in patients receiving gemcitabine [35]. The lack of significant differences in either global quality-of-life or in progression-free survival between these two groups demonstrates the need to improve existing adjuvant regimens and search for new, more effective therapeutic agents.

\section{Cisplatin}

Cis-diamminedichloridoplatinum(II) is a phase-specific chemotherapeutic drug, which fulfils its antitumor role primarily through the formation of cross-links between adjacent DNA strands and within the same strand, therefore limiting the DNA replication. Few randomised clinical trials conducted on patients treated with a combination of gemcitabine and cisplatin resulted in multifarious response rate ranging $9 \%$ to $31 \%$ but also caused toxicities like neutropaenia or thrombocytopaenia [36-39]. A trial conducted by Heinemann et al. demonstrated that median overall survival may be improved through a combining of gemcitabine and cisplatin although the results did not reach statistical significance [38]. These results, however, do not correspond with the research of other authors, which clearly expose the lack of any improvement after the addition of weekly cisplatin to gemcitabine as first-line treatment of advanced pancreatic cancer [39].

\section{Erlotinib}

Erlotinib is a reversible tyrosine kinase inhibitor, preventing initiation of signal cascade as a consequence of binding to epidermal growth factor receptor. The efficacy of EGFR inhibitors as anticancer agents has been proven, and so erlotinib has started to be utilised as a complement to gemcitabine-based therapy. A phase III trial conducted by the National Cancer Institute of Canada Clinical Trials demonstrated the superiority of treatment with erlotinib over gemcitabine alone manifested in overall survival (6.24 vs. 5.91 months), 1-year survival (23\% vs. $17 \%$ ), and progression-free survival [40]. Kelley and Ko, after analysing this and other trials involving erlotinib plus gemcitabine, identified some concerns, i.e. the possibility of an additive toxicity of this combination, the association of gender with overall survival and precarious dosage [41]. Notwithstanding the doubts, recent open-label, prospective trial clearly prove that overall survival, progression-free survival, and disease control rate may be improved after combining gemcitabine with erlotinib in comparison to gemcitabine alone, especially in patients with EGFR mutations [42].

\section{Nab-paclitaxel}

Nab-paclitaxel is a combination of the chemotherapeutic drug paclitaxel and albumin enclosed in nanoparticles. Paclitaxel represents a member of the taxane drugs family, which interacts with proteins involved in the formation of the mitotic spindle (such as tubulin) and prevents completion of mitosis. Binding it with albumin excludes the need for dissolution and supports the delivery of paclitaxel into the rapidly proliferating 
tumour cells. There is growing evidence that a combination of nab-paclitaxel and gemcitabine is superior to gemcitabine alone. Von Hoff et al. reported significantly improved overall survival, progression-free survival, and response rate after combining nab-paclitaxel with gemcitabine [43]. Also in a multinational phase III study merging nab-paclitaxel with gemcitabine resulted in prolonged overall survival mirrored in $28 \%$ reduction in the risk of death in patients with metastatic disease [44].

\section{Oxaliplatin}

Oxaliplatin is a relatively new antineoplastic drug with bidentate ligand 1,2-diaminocyclohexane in place of the two monodentate ammine ligands and bidentate oxalate group. Many pre-clinical studies have shown increased efficiency of oxaliplatin compared to cisplatin because oxaliplatin requires fewer DNA adducts to achieve an equal level of cytotoxicity [45]. Numerous clinical studies have shown the feasibility and acceptable toxicity of using oxaliplatin in pancreatic cancer therapy. However, not all of the studies showed superiority of oxaliplatin over standard used chemotherapeutics. In a randomised, multicentre phase II study on gemcitabine-refractory pancreatic cancer, a combination of S-1 and oxaliplatin (SOX) showed an advantage in response rates, but there was no significant improvement in progression-free survival (PFS) or overall survival (OS) compared with S-1 alone [46]. Despite the good tolerability, similar results were obtained in subsequent studies [47]. Combining oxaliplatin with sorafenib and capecitabine produced partial responses in patients with advanced PC and resulted in PFS of 6 months with few grade 3/4 toxicities [48]. Clinical evidence suggests that a combination of gemcitabine and oxaliplatin is superior to gemcitabine alone in advanced pancreatic carcinoma. It is also proven that this combination can produce radiosensitisation equal to or greater than gemcitabine alone [49]. This demonstrates the great potential for the use of oxaliplatin not only in adjuvant and neoadjuvant therapy, but also in palliative treatment.

\section{FOLFIRINOX}

FOLFIRINOX (FFX) regimen includes a combination of four chemotherapeutics: folinic acid (leucovorin), 5-FU, irinotecan, and oxaliplatin. It was applied for the first time in 2010 as a new treatment option for patients with metastatic pancreatic cancer [50]. Results from the PRODIGE 4/ACCORD 11 randomised trial demonstrate superiority of FFX over gemcitabine for metastatic pancreatic cancer reflected in median overall survival (11.1 months vs. 6.8 months in the gemcitabine group), median progression-free survival (6.4 months vs. 3.3 months), and objective response rate (31.6\% in the FOLFIRINOX group vs. 9.4\% in the gemcitabine group). Furthermore, therapy with FFX also had a substantial impact on improving the quality of life - a definitive degradation of the quality of life at 6 months concerned $31 \%$ of the patients in the FOLFIRINOX group compared with $66 \%$ in the gemcitabine group [51]. FFX also is proven to be a well-tolerated component of neoadjuvant therapy combined with chemoradiation [52].

\section{Radiology in pancreatic cancer}

Patients with locally advanced pancreatic cancer are not eligible for resection by definition. For these patients, treatment options are limited, and the standard is chemotherapy (CT) or a combination of chemotherapy and radiation (CRT). Statistically, 20\% to $80 \%$ of patients diagnosed with pancreatic cancer receive radiation therapy during the whole treatment [53]. For these patients the median survival amounts only to just 10 to 20 months $[54,55]$. Although radiotherapy improves overall survival and decreases the risk of neoplasm recurrence, it should be applied with caution with a view to side effects, including the development of other cancer [56]. In a systematic analysis of 15 cancer sites treated routinely with radiotherapy in a cohort including 647,672 adult 5-year survivors 60,271 (9\%) developed a second solid cancer, of which $95 \%$ could be related to radiotherapy [56]. Side effects are dependent on the duration of treatment and the radiation dose, and usually include chronical fatigue, nausea, and diarrhoea. High risk of disease recurrence after surgery makes adjuvant therapy an important part of pancreatic cancer regimen. This type of treatment, however, causes a lot of controversy due to conflicting research results. In a phase III trial of the EORTC Gastrointestinal Tract Cancer Cooperative Group, 218 patients with pancreatic head and periampullary cancers were randomised to compare survival after radiotherapy and 5-FU treatment versus observation alone after surgery. The results of the trial indicate that combined therapy is well tolerated and may bring advantages in survival. However, the benefit in median survival was not considerable (24.5 months in the treatment group vs. 19.0 months for the observation group), which may prove the illegitimacy of using chemoradiation as a routine treatment in pancreatic cancer [57]. Research conducted by the Eastern Cooperative Oncology Group, on the other hand, clearly demonstrated that radiation combined with gemcitabine is superior to gemcitabine alone for patients with locally advanced pancreatic cancer. In this trial median survival in the cohort receiving gemcitabine with radiation was 11.1 months, i.e. almost $21 \%$ higher than in the group receiving gemcitabine alone (survival amounting to 9.2 months) [55]. 
Although some results are promising, conflicting reports and high toxicity should be the basis to consider whether this method is best for patients, unless it is required for palliative purposes.

\section{Neoadjuvant therapy}

A considerable number of patients are diagnosed as "borderline resectable". For those patients it is unavoidable to improve the delivery of intend treatment. There is no protocol for neoadjuvant therapy, although it is proven that approximately one-third of patients initially diagnosed as non-resectable are amenable to surgery after neoadjuvant therapy and they have comparable survival to those who have undergone primary resection and adjuvant therapy [58]. Among acceptable therapies, the most favourable regimens are those based on FOLFIRINOX and therapy with gemcitabine comined with albumin-bound paclitaxel.

Another option that is increasingly considered as a feasible preoperative regimen is radiation. Wang and Kumar [53] have identified several advantages of neoadjuvant radiotherapy:

- Neoadjuvant RT may reduce the incidence of metastasis and improve survival.

- The use of radiation therapy prior to surgery may reduce the pathological stage of a neoplasm and increase the likelihood of complete resection.

- Radiation is better tolerated thanks to the lack of distortion caused by surgical treatment such as bowel displacement, which can lead to higher gastrointestinal toxicity.

- Surgical disruption may lead to hypoxia and cytokine stimulation resulting in a decrease in efficacy of adjuvant treatment. That could be avoided through neoadjuvant radiotherapy.

- Neoadjuvant RT may avoid unnecessary definitive surgical therapy thanks to identifying patients who are likely to develop early metastatic disease.

Combining chemotherapy with radiation may also provide rewarding effects. Preoperative therapy with full-dose gemcitabine, oxaliplatin, and radiotherapy significantly increased the number of RO resections in patients with borderline resectable disease [59].

Nevertheless, there is a clear need for including non-resectable patients into neoadjuvant protocols.

\section{Novels in pancreatic cancer therapy}

Despite the continuous search for effective treatment measures and constant improvement, curative effects and overall survival in patients diagnosed with pancreatic cancer remain disappointing. The reason may lie not only in inadequate treatment agents but also, perhaps even primarily, in difficulties with deliv- ering it to neoplasm. Therefore, current research is focused on targeted drug delivery in safe vectors such as liposomes or nanoparticles as well as exploiting new tools in the fight against cancer [60]. Modern targeted agents include toxins, oncolytic viruses, molecule inhibitors, antibodies, antisense nucleotides, small interfering RNAs, and suicide genes [60-62].

As the impact of diet and healthy lifestyle became undoubtable, the role of chemoprevention has gained tremendous importance. Among substances with proven anticancer activity the greatest attention has been attracted by products of plant origin such as resveratrol, curcumin, epigallocatechin gallate, genistein, or bensil isothiocyanate [63].

\section{Electrotherapy}

Electroporation is a modern method of supporting the penetration of macromolecules from the intercellular space into cells by application an electromagnetic field. Short pulses of high-voltage cause disorganisation of lipids and the formation of unstable space in the cell membranes. Basically, electroporation is a reversible process, but high voltage may also trigger cell lysis. In cancer therapy electroporation is used in two ways:

1) Eliminating cancer cells through irreversible electroporation.

2) Improving permeation of large molecules, like chemotherapeutic agents, through the cell membrane.

\section{Irreversible electroporation (IRE)}

Irreversible electroporation is a technique that causes total cell permeability and consequently its death [64]. Rubinsky et al. suggested that due to sparing large blood vessels scaffolds, activation of the immune system, and no scarring, IRE may be a method of treatment unresectable tumours lying near large blood vessels [65]. The great advantage of the irreversible electroporation is also its relatively short treatment delivery time [66].

A world novelty in the treatment of cancer is the NanoKnife technique. Although using this method is currently off-label, it is performed as part of a regimen in kidney, lung, prostate, and pancreas cancer treatment after notification to patients [67]. Recent studies conducted on patients aged 45 to 80 years indicate that irreversible electroporation may provide a safe and feasible method in primary local treatment of unresectable, locally advanced pancreatic adenocarcinoma [68]. Lambert et al. showed that although intraoperative IRE does not result in improved overall survival, it can ameliorate the quality of life (Karnofsky score). Therefore, electroporation constitutes a safe palliative treatment option for patients with unresectable tumours [69]. 


\section{Electrochemotherapy (ECT)}

Electrochemotherapy is a new therapeutic method reserved for cases of unresectable, advanced neoplasms, which allows the delivery of toxic agents such as bleomycin or cisplatin to tumour cells. So far it has been used in the treatment of superficial tumours, especially skin cancers, and the effects of these treatments are highly promising $[62,63]$. Moreover, recent studies have shown that ECT results in extremely high response rates ranging from $72 \%$ to $100 \%$ regardless of the histologic type and size of the tumour $[64,65]$. Although there are no large prospective, randomised studies concerning ECT application in patients with PDAC, numerous preclinical studies indicate great potential of combining electroporation with conventional chemotherapeutics and substances of natural origin, frequently galenical, such as betulinic acid, catechins, or calcium ions [70-72].

\section{Photodynamic therapy (PDT)}

Photodynamic therapy is a multistage procedure that allows for induction of selective cytotoxic reaction towards adverse cells. This technique involves the interaction of light, photosensitiser, and oxygen present in the tissues, resulting in either necrotic or apoptotic cell death [73].

The main advantages of PDT are: negligible systemic effects following selective toxicity, significantly reduced a long-term morbidity, lack of intrinsic or acquired resistance mechanisms, and sparing the cosmetic and organ function [74]. Recent research indicates that PDT restricts growth of pancreatic carcinoma and prolongs the survival of patients with unresectable tumour. However, this increase was not very prominent and further trials need to be conducted $[75,76]$.

\section{Vaccines for pancreatic cancer}

There are high expectations concerning utilisation of vaccines in cancer therapy to enhance the natural response of the immune system. Unfortunately, from 19 studies only three demonstrated a complete response, and each counted a small number of patients [77]. Despite such discouraging results, vaccines remain an open issue as a method inducing a minimum amount of adverse effects, especially for patients who have failed other treatments.

\section{Conclusions}

Over the past two decades substantial progress in diagnosis and individual therapy for pancreatic cancer has been made. Currently available literature presents many revolutionary approaches and methods of treat- ment with promising results. Among chemotherapies, combined regimens such as FOLFIRINOX and nab-paclitaxel are becoming a golden standard. There is also an increase in the importance of neoadjuvant regimens and targeted experimental therapies. Nevertheless, cancer of the pancreas, especially ductal adenocarcinoma, is still one of the most aggressive and deadly neoplasms. Our paper highlights the need to explicate novel regimens, not only to improve overall survival but also to raise the quality of life among patients grappling with this terminal disease.

\section{Acknowledgments}

This work was supported by a grant of the Medical University - grant no. Pbmn-132.

\section{Conflict of interest}

The authors declare no conflict of interest.

\section{References}

1. Krejs GJ. Pancreatic cancer: epidemiology and risk factors. Dig Dis 2010; 28: 355-8.

2. Rahib L, Smith BD, Aizenberg R, et al. Projecting cancer incidence and deaths to 2030: the unexpected burden of thyroid, liver, and pancreas cancers in the United States. Cancer Res 2014; 74: 2913-21.

3. Yu X, Zhang Y, Chen C, et al. Targeted drug delivery in pancreatic cancer. Biochim Biophys Acta 2010; 1805: 97-104.

4. Oettle H, Post S, Neuhaus P, et al. Adjuvant chemotherapy with gemcitabine vs observation in patients undergoing curative-intent resection of pancreatic cancer: a randomized controlled trial. JAMA 2007; 297: 267-77.

5. Fischer R, Breidert M, Keck T, et al. Early recurrence of pancreatic cancer after resection and during adjuvant chemotherapy. Saudi J Gastroenterol 2012; 18: 118-21.

6. Herman JM, Swartz MJ, Hsu CC, et al. Analysis of fluorouracil-based adjuvant chemotherapy and radiation after pancreaticoduodenectomy for ductal adenocarcinoma of the pancreas: results of a large, prospectively collected database at the Johns Hopkins Hospital. J Clin Oncol 2008; 26: 3503-10.

7. Chue BM. Five-year survival of metastatic pancreatic carcinoma: a study of courage and hope. Gastrointest Cancer Res 2009; 35: 208-11.

8. Malik NK, May KS, Chandrasekhar R, et al. Treatment of locally advanced unresectable pancreatic cancer: a 10-year experience. J Gastrointest Oncol 2012; 3: 326-34.

9. McWilliams RR, Maisonneuve P, Bamlet WR, et al. Risk factors for early-onset and very-early-onset pancreatic adenocarcinoma a pancreatic cancer case-control consortium (PanC4) analysis. Pancreas 2016; 45: 311-6.

10. Zheng Z, Zheng R, He Y, et al. Risk factors for pancreatic cancer in China: a multicenter case-control study. J Epidemiol 2016; 26: 64-70.

11. Li D, Morris JS, Liu J, et al. Body mass index and risk, age of onset, and survival in patients with pancreatic cancer. JAMA 2009; 301: 2553-62. 
12. Sanchez GV, Weinstein SJ, Stolzenberg-Solomon RZ. Is dietary fat, vitamin D, or folate associated with pancreatic cancer? Mol Carcinogen 2012; 51: 119-27.

13. Tramacere I, Scottie L, Jenab M, et al. Alcohol drinking and pancreatic cancer risk: a meta-analysis of the dose-risk relation. Int J Cancer 2010; 126: 1474-86.

14. Larsson SC, Wolk A. Red and processed meat consumption and risk of pancreatic cancer: meta-analysis of prospective studies. Br J Cancer 2012; 106: 603-7.

15. Klein AP, Hruban RH, Brune KA, et al. Familial pancreatic cancer. Cancer J 2001; 7: 266-73.

16. Couch FJ, Johnson MR, Rabe KG, et al. The prevalence of BRCA2 mutations in familial pancreatic cancer. Cancer Epidemiol Biomarkers Prevention 2007; 16: 342-6.

17. Giardiello FM, Brensinger JD, Tersmette AC, et al. Very high risk of cancer in familial Peutz-Jeghers syndrome. Gastroenterology 2000; 119: 1447-53.

18. Weiss FU. Pancreatic cancer risk in hereditary pancreatitis. Front Physiol 2014; 4/5: 1-5.

19. Flores K, Dinh K, Rouleau E, et al. Identification of genetic risk for pancreatic adenocarcinoma. Cancer Genet 2015; 208: 559-63.

20. Nogueira L, Freedman ND, Engels EA, et al. Gallstones, cholecystectomy, and risk of digestive system cancers. Am J Epidemiol 2014; 179: 731-9.

21. Fan Y, Hu J, Feng B, et al. Increased risk of pancreatic cancer related to gallstones and cholecystectomy: a systematic review and meta-analysis. Pancreas 2016; 45: 503-9.

22. Spanknebel K, Conlon KCP. Advances in the surgical management of pancreatic cancer. Cancer J 2001; 7: 312-23.

23. Muniraj T, Barve P. Laparoscopic staging and surgical treatment of pancreatic cancer. North Am J Med Sci 2013; 5: 1-9.

24. Sergeant G, Melloul E, Lesurtel $M$, et al. Extended lymphadenectomy in patients with pancreatic cancer is debatable. World J Surg 2013; 37: 1782-8.

25. Holzheimer RG, Mannick JA. Surgical treatment: evidencebased and problem-oriented. Zuckschwerdt, Munich 2001.

26. Lall CG, Howard TJ, Skandarajah A, et al. New concepts in staging and treatment of locally advanced pancreatic head cancer Am J Roentgenol 2007; 189: 1044-50.

27. Wagner $M$, Redaelli $C$, Lietz $M$, et al. Curative resection is the single most important factor determing outcome in patients with pancreatic adenocarcinoma. Br J Surg 2004; 91: 586-94.

28. Longley DB, Harkin DP, Johnston PG. 5-fluorouracil: mechanisms of action and clinical strategies. Nature Rev Cancer 2003; 3: 330-8.

29. Decaprio JA, Mayer RJ, Gonin R, Arbuck SG. Fluorouracil and high dose leucovorin in previously untreated patients with advanced adenocarcinoma of the pancreas - result of phase-II trial. J Clin Oncol 1991; 9: 2128-33.

30. Kulke MH. Recent developments in the pharmacological treatment of advanced pancreatic cancer. Exp Opin Investig Drugs 2003; 12: 983-92.

31. Chu QSC, Hammond LA, Schwartz G, et al. Phase I and pharmacokinetic study of the oral fluoropyrimidine S-1 on a oncedaily-for-28-day schedule in patients with advanced malignancies. Clin Cancer Res 2004; 10: 4913-21.

32. Kim YJ, Lee WJ, Woo SM, et al. Comparison of capecitabine and 5-fluorouracil in chemoradiotherapy for locally advanced pancreatic cancer. Radiat Oncol 2013; 8: 160.
33. Burris HA, Moore MJ, Andersen J, et al. Improvements in survival and clinical benefit with gemcitabine as first-line therapy for patients with advanced pancreas cancer: a randomized trial. J Clin Oncol 1997; 15: 2403-13.

34. Teague A, Lim KH, Wang-Gillam A. Advanced pancreatic adenocarcinoma: a review of current treatment strategies and developing therapies. Ther Adv Med Oncol 2015; 7: 68-84.

35. Neoptolemos JP, Stocken DD, Bassi C, et al. Adjuvant chemotherapy with fluorouracil plus folinic acid vs gemcitabine following pancreatic cancer resection: a randomized controlled trial. JAMA 2010; 304: 1073-81.

36. Brodowicz T, Wolfram RM, Kostler WJ, et al. Phase II study of gemcitabine in combination with cisplatin in patients with locally advanced and/or metastatic pancreatic cancer. Anticancer Drugs 2000; 11: 623-8.

37. Cascinu S, Labianca R, Catalano V, et al. Weekly gemcitabine and cisplatin chemotherapy: a well-tolerated but ineffective chemotherapeutic regimen in advanced pancreatic cancer patients. A report from the Italian Group for the Study of Digestive Tract Cancer (GISCAD). Ann Oncol 2003; 14: 205-8.

38. Heinemann V, Quietzsch D, Gieseler F, et al. Randomized phase III trial of gemcitabine plus cisplatin compared with gemcitabine alone in advanced pancreatic cancer. J Clin Oncol 2006; 24: 3946-52.

39. Colucci G, Labianca R, Di Costanzo F, et al. Randomized phase III trial of gemcitabine plus cisplatin compared with single-agent gemcitabine as first-line treatment of patients with advanced pancreatic cancer: the GIP-1 Study. J Clin Oncol 2010; 28: 1645-51.

40. Moore MJ, Goldstein D, Hamm J, et al. Erlotinib plus gemcitabine compared with gemcitabine alone in patients with advanced pancreatic cancer: a phase III trial of the National Cancer Institute of Canada clinical trials group. J Clin Oncol 2007; 25: 1960-6.

41. Kelley RK, Ko AH. Erlotinib in the treatment of advanced pancreatic cancer. Biologics 2008; 2: 83-95.

42. Wang JP, Wu CY, Yeh YC, et al. Erlotinib is effective in pancreatic cancer with epidermal growth factor receptor mutations: a randomized, open-label, prospective trial. Oncotarget 2015; 6: 18162-73.

43. Von Hoff DD, Ervin T, Arena FP, et al. Increased survival in pancreatic cancer with nab-Paclitaxel plus gemcitabine. N Engl J Med 2013; 369: 1691-703.

44. Hoy S. Albumin-bound paclitaxel: a review of its use for the first-line combination treatment of metastatic pancreatic cancer. Drugs 2014; 74: 1757-68.

45. Mani S, Graham MA, Bregman DB, et al. Oxaliplatin: a review of evolving concepts. Cancer Investig 2002; 20: 246.

46. Ohkawa S, Okusaka T, Isayama H, et al. Randomised phase II trial of S-1 plus oxaliplatin vs S-1 in patients with gemcitabine-refractory pancreatic cancer. Br J Cancer 2015; 112: 1428-34.

47. Takahara $\mathrm{N}$, Isayama $\mathrm{H}$, Nakai $\mathrm{Y}$, et al. A retrospective study of S-1 and oxaliplatin combination chemotherapy in patients with refractory pancreatic cancer. Cancer Chemother Pharmacol 2013; 72: 985-90.

48. Makielski RJ, Lubner SJ, Mulkerin DL, et al. A phase II study of sorafenib, oxaliplatin, and 2 days of high-dose capecitabine 
in advanced pancreas cancer. Cancer Chemother Pharmacol 2015; 76: 317-23.

49. Morgan MA, Meirovitz A, Davis MA, et al. Radiotherapy combined with gemcitabine and oxaliplatin in pancreatic cancer cells. Translat Oncol 2008; 1: 36-43.

50. Conroy T, Gavoille C, Samalin E, et al. The role of the FOLFIRINOX regimen for advanced pancreatic cancer. Curr Oncol Rep 2013; 15: 182-9.

51. Conroy T, Desseigne F, Ychou M, et al. FOLFIRINOX versus gemcitabine for metastatic pancreatic cancer. N Engl J Med 2011; 364: 1817-25.

52. Nanda RH, El-Rayes B, Maithel SK, Landry J. Neoadjuvant modified FOLFIRINOX and chemoradiation therapy for locally advanced pancreatic cancer improves resectability. J Surg Oncol 2015; 111: 1028-34.

53. Wang F, Kumar P. The role of radiotherapy in management of pancreatic cancer. J Gastrointest Oncol 2011; 2: 157-67.

54. Neoptolemos JP, Cunningham D, Friess H, et al. Adjuvant therapy in pancreatic cancer: historical and current perspectives. Ann Oncol 2003; 14: 675-92.

55. Loehrer PJ Jr, Feng Y, Cardenes H, et al. Gemcitabine alone versus gemcitabine plus radiotherapy in patients with locally advanced pancreatic cancer: an Eastern Cooperative Oncology Group Trial. J Clin Oncol 2011; 29: 4105-12.

56. de Gonzalez AB, Curtis RE, Kry SF, et al. Proportion of second cancers attributable to radiotherapy treatment in adults: a cohort study in the US SEER cancer registries. Lancet Oncol 2011; 12: 353-60.

57. Klinkenbijl JH, Jeekel J, Sahmoud T, et al. Adjuvant radiotherapy and 5-fluorouracil after curative resection of cancer of the pancreas and periampullary region - phase III trial of the EORTC Gastrointestinal Tract Cancer Cooperative Group. Ann Surg 1999; 230: 776-82.

58. Gillen S, Schuster T, zum Bueschenfelde CM, et al. Preoperative/neoadjuvant therapy in pancreatic cancer: a systematic review and meta-analysis of response and resection percentages. Plos Med 2010; 7: e1000267.

59. Kim EJ, Ben-Josef E, Herman JM, et al. A multi-institutional phase 2 study of neoadjuvant gemcitabine and oxaliplatin with radiation therapy in patients with pancreatic cancer Cancer 2013; 119: 2692-700.

60. Yu X, Zhang Y, Chen C, et al. Targeted drug delivery in pancreatic cancer. Biochim Biophys Acta 2010; 1805: 97-104.

61. De Jong WH, Borm PJA. Drug delivery and nanoparticles: applications and hazards. Int J Nanomed 2008; 3: 133-49.

62. Huang C, Li M, Chen C, Yao Q. Small interfering RNA therapy in cancer: mechanism, potential targets, and clinical applications. Exp Opin Therap Targets 2008; 12: 637-45.

63. Singh D, Upadhyay G, Srivastava RK, Shankar S. Recent advances in pancreatic cancer: biology, treatment, and prevention. Biochim Biophys Acta 2015; 1856: 13-27.

64. Rubinsky B. Technology in cancer research and treatment. Technol Cancer Res Treat 2007; 6: 255-9.

65. Rubinsky B, Onik G, Mikus P. Irreversible electroporation: a new ablation modality - clinical implications. Technol Cancer Res Treat 2007; 6: 37-48.

66. Jiang C, Davalos RV, Bischof JC. A review of basic to clinical studies of irreversible electroporation therapy. IEEE Trans Biomed Eng 2015; 62: 4-20.
67. Narayanan G. Irreversible electroporation for treatment of liver cancer. Gastroenterol Hepatol 2011; 7: 313-6.

68. Martin RCG, McFarland K, Ellis S, Velanovich V. Irreversible electroporation therapy in the management of locally advanced pancreatic adenocarcinoma. J Am Coll Surg 2012; 215: 361-9.

69. Lambert L, Horejs J, Krska Z, et al. Treatment of locally advanced pancreatic cancer by percutaneous and intraoperative irreversible electroporation: general hospital cancer center experience. Neoplasma 2016; 63: 269-73.

70. Saczko J, Pilat J, Bar J, et al. Metastatic cancers treated by electrochemotherapy with cisplatin and Betulinic acid - in vitro study. Int J Mol Med 2014; 34: S65-S.

71. Fang JY, Hung CF, Hwang TL, Wong WW. Transdermal delivery of tea catechins by electrically assisted methods. Skin Pharmacol Physiol 2006; 19: 28-37.

72. Zielichowska A, Daczewska M, Saczko J, et al. Applications of calcium electroporation to effective apoptosis induction in fibrosarcoma cells and stimulation of normal muscle cells. Bioelectrochemistry 2016; 109: 70-8.

73. Saczko J, Choromańska A, Rembiałkowska N, et al. Oxidative modification induced by photodynamic therapy with Photofrin ${ }^{\prime \prime}$ and 2-methoxyestradiol in human ovarian clear carcinoma (OvBH-1) and human breast adenocarcinoma (MCF-7) cells. Biomed Pharmacother 2015; 71: 30-6.

74. Agostinis P, Berg K, Cengel KA, et al. Photodynamic therapy of cancer: an update. CA Cancer J Clin 2011; 61: 250-81.

75. Fan BG, Andren-Sandberg A. Photodynamic therapy for pancreatic cancer. Pancreas 2007; 34: 385-9.

76. Bown SG, Rogowska AZ, Whitelaw DE, et al. Photodynamic therapy for cancer of the pancreas. Gut 2002; 50: 549-57.

77. Mizuguchi T, Torigoe T, Satomi F, et al. Trials of vaccines for pancreatic ductal adenocarcinoma: is there any hope of an improved prognosis? Surg Today 2016; 46: 139-48.

Received: 12.10 .2015

Accepted: 1.07.2016 